\title{
Dark matter density profile and galactic metric in Eddington-inspired Born-Infeld gravity
}

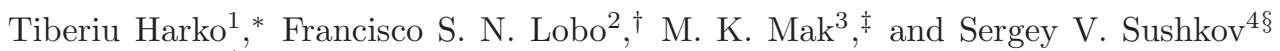 \\ ${ }^{1}$ Department of Mathematics, University College London, \\ Gower Street, London WC1E 6BT, United Kingdom \\ ${ }^{2}$ Centro de Astronomia e Astrofísica da Universidade de Lisboa, \\ Campo Grande, Ed. C8 1749-016 Lisboa, Portugal \\ ${ }^{3}$ Department of Computing and Information Management, \\ Hong Kong Institute of Vocational Education, Chai Wan, Hong Kong, and \\ ${ }^{4}$ Institute of Physics, Kazan Federal University, Kremlevskaya Street 18, Kazan 420008, Russia
}

(Dated: September 24, 2018)

\begin{abstract}
We consider the density profile of pressureless dark matter in Eddington-inspired Born-Infeld (EiBI) gravity. The gravitational field equations are investigated for a spherically symmetric dark matter galactic halo, by adopting a phenomenological tangential velocity profile for test particles moving in stable circular orbits around the galactic center. The density profile and the mass distribution, as well as the general form of the metric tensor is obtained by numerically integrating the gravitational field equations, and in an approximate analytical form by using the Newtonian limit of the theory. In the weak field limit the dark matter density distribution is described by the Lane-Emden equation with polytropic index $n=1$, and is non-singular at the galactic center. The parameter $\kappa$ of the theory is determined so that that the theory could provide a realistic description of the dark matter halos. The gravitational properties of the dark matter halos are also briefly discussed in the Newtonian approximation.
\end{abstract}

PACS numbers: $04.50 . \mathrm{Kd}, 04.20 . \mathrm{Cv}$

\section{INTRODUCTION.}

Despite numerous observational and experimental attempts, up to now the elusive dark matter particles have evaded detection. Great hopes in gaining more insights into the nature of dark matter are related to the Alpha Magnetic Spectrometer (AMS-02) collaboration results, which have just been released [1]. The AMS experiment has determined the positron fraction in cosmic rays with extremely high precision. The positron fraction rises continuously from $\sim 5 \mathrm{GeV}$ up to $\sim 350 \mathrm{GeV}$, while the slope becomes flat above $\sim 100 \mathrm{GeV}$ [1]. In order to explain the AMS results, dark matter is still an attractive candidate. In fact, dark matter annihilation into $\tau^{+} \tau^{-}$final states, which results in a soft positron spectrum can account for the AMS-02 data quite well. Other channels for dark matter annihilation/decay into multiple $\mu$ or $\tau$ leptons can also reproduce the AMS-02 data [2]. However, presently, the only convincing evidence, based on the flat rotation galactic curves and the virial mass discrepancy in clusters of galaxies for the existence of dark matter, is gravitational [3]- 7]].

In standard general relativity the coupling between matter and gravity is given by a proportionality relation between the stress-energy tensor and the geometry. Although both the stress-energy tensor and the Einstein

\footnotetext{
*Electronic address: t.harko@ucl.ac.uk

${ }^{\dagger}$ Electronic address: flobo@cii.fc.ul.pt

‡Electronic address: mkmak@vtc.edu.hk

$\S$ Electronic address: sergey`sushkov@mail.ru
}

tensor are divergenceless, there is no obvious reason why the matter-gravity coupling should be linear $[8,[9]$. On the other hand, modified theories of gravity usually affect the vacuum dynamics, yet keep the matter-gravity coupling linear. Recently, to provide matter-gravity coupling modifications, based on the Eddington gravity action [10], and Born-Infeld nonlinear electrodynamics [11], the Eddington-inspired Born-Infeld (EiBI) theory has been proposed [12, 13]. The EiBI theory coupled to a perfect fluid reduces to standard general relativity coupled to a nonlinearly modified perfect fluid, leads to an ambiguity between modified coupling and a modified equation of state [14, 15]. The theory has interesting cosmological consequences, leading to the possibility of avoiding cosmological singularities 16]. The structure of the neutron and quark stars for different equations of state of the dense matter were investigated in [17]. However, the EiBI theory is reminiscent of the Palatini gravity formulation, and it shares the same pathologies, such as curvature singularities at the surface of polytropic stars [18].

It is the purpose of this Letter to investigate the properties of dark matter halos in the EiBI gravity theory. By assuming a spherically symmetric pressureless dark matter halo, and a very general tangential rotation curve profile, the gravitational field equations of the EiBI model are exactly solved numerically, and the behavior of the dimensionless density and mass profiles, as well as the metric tensor coefficients are explicitly obtained. The dark matter distribution has a very sharp boundary that defines the radius of the dark matter distribution. In order to obtain an analytic representation of the dark matter halo properties the gravitational field equations 
describing the dark matter distribution are solved to a first order approximation of the coupling constant $\kappa$. The dark matter density profile and the galactic metric in the dark matter halo is explored, and explicitly obtained, and the respective gravitational properties are also briefly discussed in the Newtonian approximation.

The present paper is organized as follows. The Eddington-Inspired Born-Infeld gravity is briefly reviewed in Section [II. The basic field equations describing the galactic dark matter halo properties are written down in Section III In Section IV we solve numerically the field equations describing dark matter halos, and obtain the density and the mass distributions, as well as the metric coefficients. An approximate solution of the field equations is obtained, and its properties are discussed in Section $\nabla$. We discuss and conclude our results in Section VI.

\section{EDDINGTON-INSPIRED BORN-INFELD GRAVITY}

The starting point of the EiBI theory is the gravitational action $S$, given by [13],

$$
\begin{aligned}
S= & \frac{1}{8 \pi} \frac{1}{\kappa} \int d^{4} x\left(\sqrt{-\left|g_{\mu \nu}+\kappa R_{\mu \nu}\right|}-\lambda \sqrt{-g}\right)+ \\
& S_{M}\left[g, \Psi_{M}\right]
\end{aligned}
$$

where $\lambda \neq 0$ is a dimensionless parameter, and $\kappa$ is a parameter with inverse dimension to that of the cosmological constant $\Lambda . R_{\mu \nu}$ is the symmetric part of the Ricci tensor, and is constructed solely from the connection $\Gamma_{\beta \gamma}^{\alpha}$. The matter action $S_{M}$ depends only on the metric $g_{\mu \nu}$ and the matter fields $\Psi_{M}$. The determinant of the tensor $g_{\mu \nu}+\kappa R_{\mu \nu}$ is denoted by $\left|g_{\mu \nu}+\kappa R_{\mu \nu}\right|$. In the limit $\kappa \rightarrow 0$, the action Eq. (11) recovers the EinsteinHilbert action with $\lambda=\Lambda \kappa+1$. However, in the present paper, we consider only asymptotic flat solutions, and hence take $\lambda=1$. Therefore the cosmological constant vanishes, and the remaining parameter $\kappa$ plays the determining role for the description of the physical behavior of various cosmological and stellar scenarios. Several constraints on the value and the sign of the parameter $\kappa$ have been obtained from solar observations, Big Bang nucleosynthesis, and the existence of neutron stars in [13, 19 22]. The structure of compact stars in EiBI theory has been investigated by several authors [19, 23, 24]. In particular, for cases with positive $\kappa$, effective gravitational repulsion prevails, leading to the existence of pressureless stars (stars made of non-interacting particles which provide interesting models for self-gravitating dark matter) and increases in the mass limits of compact stars 1924 .

In the EiBI theory the metric $g_{\mu \nu}$ and the connection $\Gamma_{\beta \gamma}^{\alpha}$ are treated as independent fields. By varying the action (11) with respect to the connection $\Gamma_{\beta \gamma}^{\alpha}$, and with respect to the real metric $g_{\mu \nu}$, yield the following field equations $q_{\mu \nu}=g_{\mu \nu}+\kappa R_{\mu \nu}$ and $q^{\mu \nu}=\tau\left(g^{\mu \nu}-8 \pi \kappa T^{\mu \nu}\right)$, respectively. The auxiliary metric $q_{\mu \nu}$ is related to the connection by $\Gamma_{\beta \gamma}^{\alpha}=\frac{1}{2} q^{\alpha \sigma}\left(\partial_{\gamma} q_{\sigma \beta}+\partial_{\beta} q_{\sigma \gamma}-\partial_{\sigma} q_{\beta \gamma}\right)$, and $\tau$ is defined as $\tau=\sqrt{g / q}$. If the stress-energy tensor $T^{\mu \nu}$ vanishes, then the real metric $g_{\mu \nu}$ is equal to the apparent metric $q_{\mu \nu}$. Hence, in vacuum the EiBI theory is equivalent to standard general relativity. The stress-energy tensor $T^{\mu \nu}$ satisfies the conservation equations given by $\nabla_{\mu} T^{\mu \nu}=0$, where the covariant derivative $\nabla_{\mu}$ refers to the metric $g_{\mu \nu}$.

\section{STATIC SPHERICALLY SYMMETRIC DARK MATTER HALOS IN EIBI GRAVITY}

We consider that the dark matter halo is static and spherically symmetric. Therefore the line elements for the physical metric $g_{\mu \nu}$ and for the auxiliary metric $q_{\mu \nu}$ take the forms

$$
\begin{aligned}
& g_{\mu \nu} d x^{\mu} d x^{\nu}=-e^{\nu(r)} c^{2} d t^{2}+e^{\lambda(r)} d r^{2}+f(r) d \Omega^{2} \\
& q_{\mu \nu} d x^{\mu} d x^{\nu}=-e^{\beta(r)} c^{2} d t^{2}+e^{\alpha(r)} d r^{2}+r^{2} d \Omega^{2}
\end{aligned}
$$

respectively, where $\nu(r), \lambda(r), \beta(r), \alpha(r)$ and $f(r)$ are arbitrary metric functions of the radial coordinate $r$, and $d \Omega^{2}=d \theta^{2}+\sin ^{2} \theta d \phi^{2}$. The galactic halo is made up of a perfect fluid described by the standard stress-energy tensor

$$
T^{\mu \nu}=\left(\rho c^{2}+p\right) u^{\mu} u^{\nu}+p g^{\mu \nu},
$$

where $\rho, p$ and $u^{\mu}$ are the energy density, the isotropic pressure and the four velocity of the fluid, respectively, with the latter satisfying $u^{\mu} u^{\nu} g_{\mu \nu}=-1$. The system of gravitational field equations describing the dark matter halo structure yields [23, 24]

$$
\begin{array}{r}
\frac{d}{d r}\left(r e^{-\alpha}\right)=1-\frac{1}{2 \kappa}\left(2+\frac{a}{b^{3}}-\frac{3}{a b}\right) r^{2}, \\
e^{-\alpha}\left(1+r \frac{d \beta}{d r}\right)=1+\frac{1}{2 \kappa}\left(\frac{1}{a b}+\frac{a}{b^{3}}-2\right) r^{2}, \\
e^{\beta}=\frac{e^{\nu} b^{3}}{a}, \quad e^{\alpha}=e^{\lambda} a b, \quad f=\frac{r^{2}}{a b},
\end{array}
$$

where we have defined the arbitrary functions $a(r)$ and $b(r)$ as

$$
a=\sqrt{1+8 \pi \kappa \frac{G}{c^{2}} \rho},
$$

and

$$
b=\sqrt{1-8 \pi \kappa \frac{G}{c^{4}} p},
$$

respectively. In the $g$-metric the conservation of the stress-energy tensor yields the result

$$
\frac{d \nu}{d r}=-\frac{2}{p+\rho c^{2}} \frac{d p}{d r}=\frac{4 b}{a^{2}-b^{2}} \frac{d b}{d r} .
$$


The existence of dark matter at the galactic scale is inferred from the study of the rotational velocities of massive test particles (hydrogen clouds) around the galactic center. The Lagrangian $\mathcal{L}$ for a massive test particle reads

$$
\mathcal{L}=\frac{1}{2}\left(-e^{\nu} c^{2} \dot{t}^{2}+e^{\lambda} \dot{r}^{2}+r^{2} \dot{\Omega}^{2}\right),
$$

where the overdot denotes differentiation with respect to the affine parameter $s$. By defining the tangential velocity $v_{\text {tg }}$ of a test particle, as measured in terms of the proper time [25], that is, by an observer located at a given point, as $v_{t g}^{2}=e^{-\nu} r^{2}(d \Omega / d t)^{2}$, we obtain the expression of $v_{t a}^{2}$ for a test particle in a stable circular orbit as [26] - 30]

$$
\frac{v_{t g}^{2}}{c^{2}}=\frac{1}{2} r \nu^{\prime}
$$

For $v_{t g}^{2}$ we assume the simple empirical dark halo rotational velocity law 31]

$$
v_{t g}^{2}=v_{0 \infty}^{2} \frac{\left(r / r_{o p t}\right)^{2}}{\left(r / r_{o p t}\right)^{2}+r_{0}^{2}}
$$

where $r_{o p t}$ is the optical radius containing $83 \%$ of the galactic luminosity. The parameter $r_{0}$, defined as the ratio of the halo core radius and $r_{\text {opt }}$, and the terminal velocity $v_{\infty}$ are functions of the galactic luminosity $L$. For spiral galaxies $r_{0}=1.5\left(L / L_{*}\right)^{1 / 5}$ and $v_{\infty}^{2}=v_{o p t}^{2}\left(1-\beta_{*}\right)\left(1+r_{0}^{2}\right)$, where $v_{o p t}=v_{t g}\left(r_{o p t}\right)$, and $\beta_{*}=0.72+0.44 \log _{10}\left(L / L_{*}\right)$, with $L_{*}=10^{10.4} L_{\odot}$. For $r \rightarrow 0, v_{t g} \rightarrow 0$, while for $r / r_{\text {opt }} \gg r_{0}, v_{t g} \rightarrow v_{0 \infty}$. In the following we denote $v_{\infty}$ as

$$
v_{\infty}^{2}=\frac{v_{0 \infty}^{2}}{c^{2}} .
$$

Therefore, the most general static and spherically symmetric metric of the dark matter halo can be written as

$$
d s^{2}=-e^{\nu_{0}}\left[\left(\frac{r}{r_{o p t}}\right)^{2}+r_{0}^{2}\right]_{\infty}^{v_{\infty}^{2}} d t^{2}+e^{\lambda(r)} d r^{2}+r^{2} d \Omega^{2},
$$

where $e^{\nu_{0}}$ is an arbitrary constant of integration.

In the Newtonian limit the $g_{t t}$ component of the metric tensor is given by $e^{\nu} \approx 1+2 \Phi_{N}$, where $\Phi_{N}$ is the Newtonian gravitational potential satisfying the Poisson equation $\Delta \Phi_{N}=4 \pi \rho$ [25]. In the constant velocity region the mass $M(r)$ of the dark matter and the energy density $\rho$ vary with the distance as $M(r)=v_{\mathrm{tg}}^{2} r$ and $\rho=v_{\mathrm{tg}}^{2} / 4 \pi r^{2}$, respectively.

\section{THE DARK MATTER DENSITY PROFILE AND THE GALACTIC METRIC IN EIBI GRAVITY}

In the present Section we will restore the normal astrophysics units in all equations. In order to study the extra-galactic motion of massive test particles in EiBI gravity, we assume that the motion takes place in a cosmic medium that satisfies the $\left(8 \pi G \kappa / c^{4}\right) p \ll 1$. Therefore we immediately obtain $b \approx 1$, and hence the dark matter properties can be described by the effective matter density $\rho$ only, which determines the function $a$. For a galactic metric of the form given by Eq. (15) we obtain

$$
\begin{aligned}
e^{\beta} & =\frac{e^{\nu_{0}}\left[\left(r / r_{o p t}\right)^{2}+r_{0}^{2}\right]_{\infty}^{v_{\infty}^{2}}}{\sqrt{1+8 \pi \kappa \rho}} \\
\beta^{\prime} & =2 v_{\infty}^{2} \frac{r / r_{o p t}^{2}}{\left(r / r_{o p t}\right)^{2}+r_{0}^{2}}-\frac{4 \pi \kappa G}{c^{2}} \frac{\rho^{\prime}}{1+\left(8 \pi G \kappa / c^{2}\right) \rho},
\end{aligned}
$$

respectively.

Then, by using a series expansion of $a$ and $\beta^{\prime}$ to first order of $\kappa$, we obtain

$$
\begin{aligned}
& \left(1+8 \pi \frac{G}{c^{2}} \kappa \rho\right)^{-1 / 2}+\sqrt{1+8 \pi \frac{G}{c^{2}} \kappa \rho}-2 \approx 0, \\
& 2+\sqrt{1+8 \pi \frac{G}{c^{2}} \kappa \rho}-3\left(1+8 \pi \frac{G}{c^{2}} \kappa \rho\right)^{-1 / 2} \approx 16 \pi \frac{G}{c^{2}} \kappa \rho,
\end{aligned}
$$

and

$$
\beta^{\prime}=2 \frac{v_{\infty}^{2}}{\left(r / r_{o p t}\right)^{2}+r_{0}^{2}} \frac{r}{r_{o p t}^{2}}-\frac{4 \pi \kappa G}{c^{2}} \rho^{\prime} .
$$

Therefore the field equations Eqs. (5)-(6) become

$$
\frac{d}{d r}\left(r e^{-\alpha}\right)=1-8 \pi \rho r^{2}
$$

and

$$
r e^{-\alpha}=\frac{1}{1 / r+2 v_{\infty}^{2}\left(r / r_{o p t}^{2}\right) /\left[\left(r / r_{o p t}\right)^{2}+r_{0}^{2}\right]-4 \pi \kappa \rho^{\prime}},
$$

respectively.

\section{A. The density profile and the metric of the dark matter halos in EiBI gravity}

In order to obtain a simpler form of Eqs. (21) and (22) we introduce a set of dimensionless variables $(\eta, \theta)$ defined as

$$
r=r_{o p t} \eta, \quad \rho=\frac{c^{2}}{8 \pi G r_{o p t}^{2}} \theta
$$

Hence Eqs. (21) and (22) can be written in a dimensionless form as

$$
\frac{d}{d \eta}\left(\eta e^{-\alpha}\right)=1-\theta \eta^{2},
$$


and

$$
\eta e^{-\alpha}=\left(\frac{1}{\eta}+2 v_{\infty}^{2} \frac{\eta}{\eta^{2}+r_{0}^{2}}-k_{0} \frac{d \theta}{d \eta}\right)^{-1},
$$

respectively, where we have denoted

$$
k_{0}=\frac{\kappa}{2 r_{o p t}^{2}} .
$$

By taking the derivative with respect to $\eta$ of Eq. (25), with the use of Eq. (24) we obtain the following equation describing the behavior of the dark matter density in the EiBi gravity

$$
\begin{aligned}
\theta^{\prime \prime}= & -\kappa_{0}\left(\eta^{2} \theta-1\right) \theta^{\prime 2}-\frac{\left[r_{0}^{2}+\eta^{2}\left(2 v_{\infty}^{2}+1\right)\right]^{2}}{\kappa_{0}\left(\eta^{2}+r_{0}^{2}\right)^{2}} \theta+ \\
& \frac{2\left(\eta^{2} \theta-1\right)\left(\eta^{2}+r_{0}^{2}+2 \eta^{2} v_{\infty}^{2}\right)}{\eta\left(\eta^{2}+r_{0}^{2}\right)} \theta^{\prime}+ \\
& \frac{6 v_{\infty}^{2}}{\kappa_{0}\left(\eta^{2}+r_{0}^{2}\right)}+\frac{4 \eta^{2}\left(v_{\infty}^{2}-1\right) v_{\infty}^{2}}{\kappa_{0}\left(\eta^{2}+r_{0}^{2}\right)^{2}}
\end{aligned}
$$

Equation (27) must be integrated with the initial conditions $\theta(0)=\theta_{c}$, and $\theta^{\prime}(0)$, respectively. The second of Eqs. (23) provides

$$
\theta_{c}=\frac{8 \pi G r_{o p t}^{2}}{c^{2}} \rho_{c} .
$$

In the following we will fix the dark matter halo parameters so that $r_{\text {opt }}=5 \mathrm{kpc}=1.5 \times 10^{22} \mathrm{~cm}$, and $\rho_{c}=10^{-24}$ $\mathrm{g} / \mathrm{cm}^{3}$. This gives $\theta_{c}=4.19 \times 10^{-7}$. For the tangential velocity in the constant velocity region we adopt the value $v_{\infty}=100 \mathrm{~km} / \mathrm{s}$, while for $r_{0}$ we take the value $r_{0}=1$.

The variation of the dimensionless dark matter density profile $\theta$ as a function of $\eta$, and for different values of $\kappa_{0}$, is represented in Fig. 1. As one can see from Fig. 11 in the EiBI gravity the dark matter halos have a sharp boundary $\eta_{S}$, which defines the radius $R_{D M}$ of the galaxy. For the numerical values of the parameter $\kappa_{0}$ considered in the numerical analysis, we obtain $R_{D M}=\eta_{S} r_{o p t} \approx 12.5-17.5 \mathrm{kpc}$, a value which is reasonable from an astrophysical point of view. Hence, in order to explain the observed properties of the dark matter halos the parameter $\kappa$ of the theory must have a value of the order

$$
\kappa \approx 2 r_{o p t}^{2} \kappa_{0} \approx 2.25 \times 10^{44} \mathrm{~cm}^{2} .
$$

The variation of the metric coefficient $e^{-\lambda}=$ $\sqrt{1+2 \kappa_{0} \theta} e^{-\alpha}$ is represented in Fig. 2

The mass of the dark matter halo $M_{D m}(r)$ is given by

$$
\frac{G M_{D M}(r)}{c^{2}}=\frac{r}{2}\left(1-\sqrt{1+8 \pi \frac{G}{c^{2}} \kappa \rho e^{-\alpha}}\right) .
$$

By introducing the dimensionless dark matter mass $m(\eta)$, defined as

$$
M_{D M}(r)=\frac{c^{2} r_{o p t}}{G} m(\eta)
$$

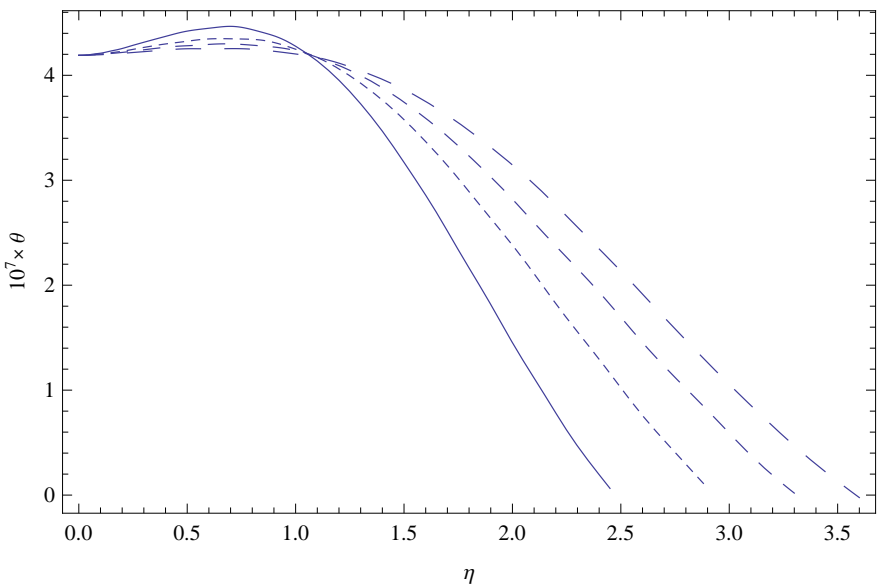

FIG. 1: Variation of the dimensionless dark matter density profile $\theta$ as a function of the dimensionless radial coordinate $\eta$ for different values of $\kappa_{0}: \kappa_{0}=0.3$ (solid curve), $\kappa_{0}=0.5$ (dashed curve), $\kappa_{0}=0.7$ (short dashed curve), and $\kappa_{0}=1$ (long dashed curve), respectively. The initial conditions are $\theta(0)=4.19 \times 10^{-7}$ (corresponding to $r_{o p t}=5 \mathrm{kpc}$ and a dark matter central density $\left.\rho_{c}=10^{-24} \mathrm{~g} / \mathrm{cm}^{3}\right)$, and $\theta^{\prime}(0)=10^{-7}$. For the values of the astrophysical parameters describing the halo we have adopted the numerical values $v_{0 \infty}=100 \mathrm{~km} / \mathrm{s}$, and $r_{0}=1$, respectively.

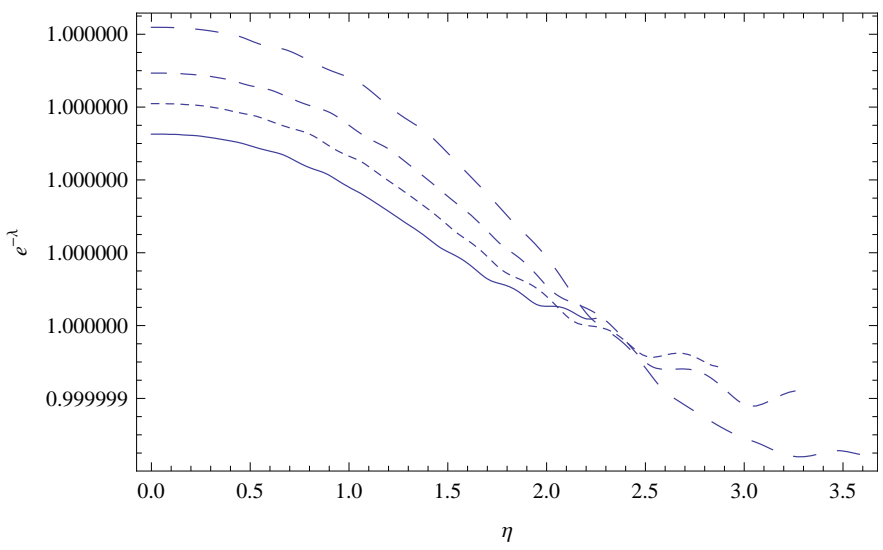

FIG. 2: Variation of the metric coefficient $e^{-\lambda}=$ $\sqrt{1+2 \kappa_{0} \theta} e^{-\alpha}$ as a function of the dimensionless radial coordinate $\eta$ for different values of $\kappa_{0}: \kappa_{0}=0.3$ (solid curve), $\kappa_{0}=0.5$ (dashed curve), $\kappa_{0}=0.7$ (short dashed curve), and $\kappa_{0}=1$ (long dashed curve), respectively. The initial conditions are $\theta(0)=4.19 \times 10^{-7}$ (corresponding to $r_{\text {opt }}=5$ $\mathrm{kpc}$ and a dark matter central density $\rho_{c}=10^{-24} \mathrm{~g} / \mathrm{cm}^{3}$ ), and $\theta^{\prime}(0)=10^{-7}$. For the values of the astrophysical parameters describing the halo we have adopted the numerical values $v_{0 \infty}=100 \mathrm{~km} / \mathrm{s}$, and $r_{0}=1$, respectively.

we obtain

$$
\begin{aligned}
m(\eta)= & \frac{\eta}{2}\left(1-\sqrt{1+2 \kappa_{0} \theta} e^{-\alpha}\right)= \\
& \eta\left(1-\frac{\sqrt{1+2 \kappa_{0} \theta}}{\frac{1}{\eta}+2 v_{\infty}^{2} \frac{\eta}{\eta^{2}+r_{0}^{2}}-k_{0} \frac{d \theta}{d \eta}}\right) .
\end{aligned}
$$




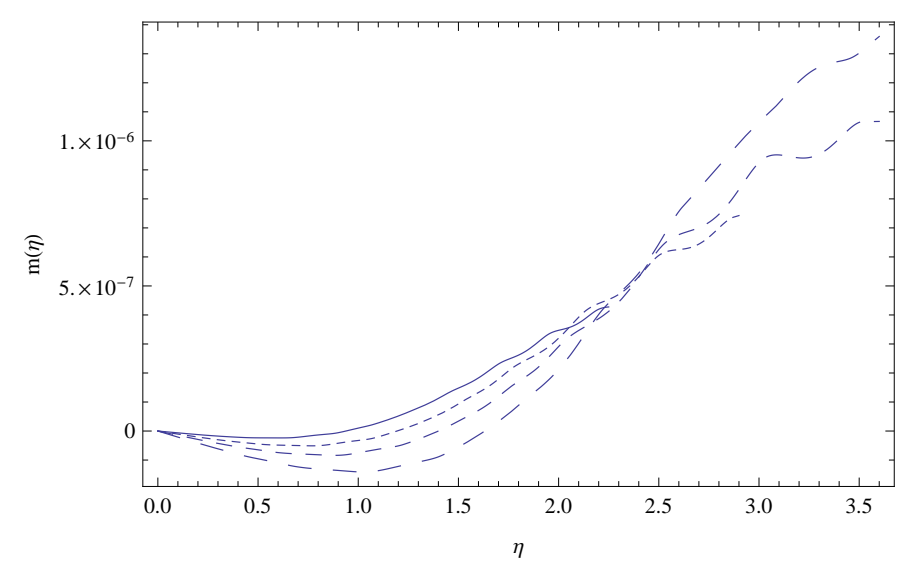

FIG. 3: Variation of the dimensionless mass profile of the dark matter halo in the EiBI gravity as a function of the dimensionless radial coordinate $\eta$ for different values of $\kappa_{0}: \kappa_{0}=0.3$ (solid curve), $\kappa_{0}=0.5$ (dashed curve), $\kappa_{0}=0.7$ (short dashed curve), and $\kappa_{0}=1$ (long dashed curve), respectively. The initial conditions are $\theta(0)=4.19 \times 10^{-7}$ (corresponding to $r_{o p t}=5 \mathrm{kpc}$ and a dark matter central density $\rho_{c}=10^{-24}$ $\left.\mathrm{g} / \mathrm{cm}^{3}\right)$, and $\theta^{\prime}(0)=10^{-7}$. For the values of the astrophysical parameters describing the halo we have adopted the numerical values $v_{0 \infty}=100 \mathrm{~km} / \mathrm{s}$, and $r_{0}=1$, respectively.

The variation of the mass density profile of the dark matter in the EiBI gravity is represented in Fig. 3.

\section{DARK MATTER HALOS IN THE NEWTONIAN LIMIT}

In the non-relativistic limit the action Eq. (11) leads to the modified Poisson equation for the gravitational potential $\Phi$, given by [13, 19],

$$
\nabla^{2} \Phi=4 \pi G \rho+G \frac{\kappa}{4} \nabla^{2} \rho
$$

For a spherically symmetric static dark matter distribution, with the use of Eq. (33), we obtain the hydrostatic equilibrium equation as [19]

$$
\frac{d p}{d r}=-\frac{G M}{r^{2}} \rho-G \frac{\kappa}{4} \rho \rho^{\prime} .
$$

In the case of dark matter halos the approximation $p=0$ gives a very good description of the galactic properties of the dark matter. By combining Eq. (34) with $p=0$ with the mass continuity equation

$$
\frac{d M}{d r}=4 \pi \rho r^{2}
$$

we obtain the following equation describing the density distribution of the dark matter halos in the Newtonian approximation of EiBI gravity,

$$
\frac{1}{r^{2}} \frac{d}{d r}\left(r^{2} \frac{d \rho^{(0)}}{d r}\right)+\frac{2}{\bar{\kappa}} \rho^{(0)}=0
$$

where we have denoted by $\rho^{(0)}$ the density in the Newtonian approximation, and $\bar{\kappa}=\kappa / 8 \pi$, respectively.

Eq. (36) is the Lane-Emden equation for the polytropic index $n=1$ [32, 33], and its non-singular solution at the center is given by

$$
\rho^{(0)}(r)=K \frac{\sin (\sqrt{2 / \bar{\kappa}} r)}{\sqrt{2 / \bar{\kappa}} r}
$$

where $K$ is an arbitrary constant of integration. Since at the center of the galactic halo the density of the dark matter is $\rho^{(0)}(0)=\rho_{0}$, it follows that $K=\rho_{0}$. In the EiBI theory the dark halo has a sharp boundary $R_{D M}$, corresponding to $\rho^{(0)}\left(R_{D M}\right)=0$, which gives $R_{D M}=\sqrt{\bar{\kappa} / 2} \pi$. Thus, the mass profile of the dark matter $M(r)=4 \pi \int_{0}^{r} \rho^{(0)}(r) r^{2} d r$ is given by

$$
M(\bar{r})=\frac{4 R_{D M}^{3}}{\pi^{2}} \rho_{0}[\sin (\bar{r})-\bar{r} \cos (\bar{r})],
$$

where $\bar{r}=\pi r / R_{D M}$ is defined for convenience.

The $q$-metric coefficient $e^{-\alpha}=1-2 G M(r) / c^{2} r$ yields

$$
\begin{aligned}
e^{-\alpha}= & 1-\frac{8 R_{D M}^{2}}{\pi \bar{r}} \rho_{0}[\sin (\bar{r})-\bar{r} \cos (\bar{r})]= \\
& 1-\frac{\bar{\rho}_{0}}{\bar{r}}[\sin (\bar{r})-\bar{r} \cos (\bar{r})],
\end{aligned}
$$

where $\bar{\rho}_{0}=8 G \rho_{0} R_{D M}^{2} / \pi c^{2}$. For the metric coefficient $e^{-\lambda}=e^{-\alpha} a$, we obtain

$$
e^{-\lambda} \approx e^{-\alpha}\left(1+4 \pi \kappa \frac{G}{c^{2}} \rho\right)=e^{-\alpha}\left[1+\frac{\bar{\rho}_{0}}{\bar{r}} \sin (\bar{r})\right],
$$

while the function $f(\bar{r})$ is given by

$$
f(\bar{r})=\frac{\left(R_{D M}^{2} / \pi^{2}\right) \bar{r}^{2}}{\sqrt{1+8 \pi \kappa \rho}} \approx \frac{R_{D M}^{2}}{\pi^{2}} \bar{r}^{2}\left[1-\frac{\bar{\rho}_{0}}{\bar{r}} \sin (\bar{r})\right] .
$$

Thus, the metric of the dark matter halo takes the form

$$
\begin{aligned}
& d s^{2}=-e^{\nu_{0}}\left[\left(\frac{R_{D M}}{\pi r_{\text {opt }}}\right)^{2} \bar{r}^{2}+r_{0}^{2}\right]_{\infty}^{v_{\infty}^{2}} c^{2} d t^{2}+ \\
& \frac{R_{D M}^{2}}{\pi^{2}} \frac{d \bar{r}^{2}}{\left[1-\frac{\bar{\rho}_{0}}{\bar{r}} \sin (\bar{r})+\bar{\rho}_{0} \cos (\bar{r})\right]\left[1-\frac{\bar{\rho}_{0}}{\bar{r}} \sin (\bar{r})\right]}+ \\
& \frac{R_{D M}^{2}}{\pi^{2}} \bar{r}^{2}\left[1-\frac{\bar{\rho}_{0}}{\bar{r}} \sin (\bar{r})\right] d \Omega^{2}
\end{aligned}
$$

The total mass of the dark matter halo is given by $M_{D M}=M\left(R_{D M}\right)=\sqrt{2} \pi^{2} \kappa^{3 / 2} \rho_{0}=(4 / \pi) \rho_{0} R_{D M}^{3}$. The mean density $\langle\rho\rangle$ of the dark matter halo is obtained as $<\rho>=3 M_{D M} / 4 \pi R_{D M}^{3}=3 \rho_{0} / \pi^{2}$.

In the Newtonian approximation the gravitational potential $V_{\text {grav }}(r)$ of the dark matter distribution in the EiBI theory is determined for $\bar{r} / \pi \leq 1$ by

$$
V_{\text {grav }}=\frac{1}{8 \pi} \int_{r}^{R_{D M}} \frac{M\left(r^{\prime}\right) d r^{\prime}}{r^{\prime 2}}=\frac{\bar{\rho}_{0}}{16 \pi \bar{r}} \sin (\bar{r}) .
$$


At small radii and for $\bar{r} / \pi \leq 1$ the potential behaves as

$$
V_{\text {grav }}(\bar{r}) \approx \frac{\bar{\rho}_{0}}{16 \pi}\left(1-\frac{1}{3 !} \bar{r}^{2}+\frac{1}{5 !} \bar{r}^{4}-\frac{1}{7 !} \bar{r}^{6}\right)+O(\bar{r})^{8} .
$$

The gravitational potential energy $U(r)$ per unit mass and inside radius $r$ of the dark matter halo yields

$$
\begin{aligned}
U= & -\frac{1}{2} \int_{0}^{r} \frac{\rho^{(0)}(r) M(r)}{r} r^{2} d r=-\frac{R_{D M}^{5} \rho_{0}^{2}}{4 \pi^{3}} \times \\
& \{2 \bar{r}[2+\cos (2 \bar{r})]-3 \sin (2 \bar{r})\} .
\end{aligned}
$$

Thus, the total potential energy of the dark matter halo is given by $U\left(R_{D M}\right)=-\left(3 \rho_{0}^{2} / 2 \pi^{2}\right) R_{D M}^{5}$.

\section{DISCUSSIONS AND FINAL REMARKS}

In the present paper, we have analyzed the dark matter density profiles, and their geometry, in the EiBI gravitational theory. As a first step in our study we have adopted a very general tangential velocity profile 31], which, together with the requirement of the motion of the test particles in stable circular orbits, allows to formulate the basic equations describing the general relativistic dark matter halos in the EiBI theory. The solution of the field equations has been obtained numerically, after reducing them to a dimensionless form.

In the Newtonian approximation of the dark matter pressure satisfying the condition $p=0$, the dark matter density profile satisfies the Lane-Emden equation with a polytropic index $n=1$ [32, 33]. Therefore the dark matter profile can be obtained in an exact analytic form, as well as the full galactic dark matter metric, in both $g$ and $q$ geometries. Similar results for pressureless stellar profiles have been obtained in [15]. The non-singular $n=1$ polytropic density profiles show the presence of an extended core, whose presence in the considered model is due to the matter-gravity coupling, and modified geometry.

In fact, in the context of extended theories of gravity, it was argued that the generic corrections to the Newtonian potential could fit data and reproduce the observational galactic dynamics [34]. These corrections are not phenomenological but arise from the weak field limit of the extended relativistic theories of gravity that predict the existence of Yukawa-like corrections to the Newtonian potential. These corrections imply that further scales have to be taken into account and that their effects should be irrelevant at local Solar System scales. Indeed, considering the modified Lane-Emden equation arising from $f(R)$ gravity, it was shown that the differences between GR and $f(R)$ gravitational potentials become evident for larger radii from the centre, so that the corrections to the potential can significantly boost the circular velocity for an extended system such as in a galaxy. In the strong field regime, such corrections could give rise to peculiar stellar structure or trigger the Jeans instability, and this issue was further explored in [35].
As follows from our numerical results, in order to obtain realistic dark matter density profiles the unique free parameter of the EiBI gravity must be of the order of $\kappa \approx 10^{44} \mathrm{~cm}^{2}$. This result is consistent with the value of the galactic halo radius obtained in the Newtonian approximation. On the other hand the studies of the structure of the compact general relativistic stars require a value of $\kappa$ of the order of $\kappa=10^{12} \mathrm{~cm}^{2}$ 17, 20 22. Moreover, for a given $\kappa$, the theory predicts a universal dark matter halo distribution. These contradictory results raise the question of the viability of the EiBI gravity as a theoretical model that could describe gravitational phenomena on all astrophysical and cosmological scales. It is interesting to note that similar constraints requiring very large values of $\kappa$ have been obtained in [18] from the requirement of the absence of strong near-surface curvature effects. For simple Newtonian configurations in which the density distribution deviates from perfect smoothness at a given cut-off surface the EiBI theory may yield surface singularities. For the case of a polytropic star with $M=1.4 M_{\odot}$ and radius $R=10^{-2} R_{\odot}$, the constraint on $\kappa$ can be formulated as $\kappa \geq 6 \times 10^{38+2 k}$ $\mathrm{cm}^{2}$, where $k$ is a parameter related to the form of the polytropic equation of state that provides a reliable description of the matter close to the surface of the star.

In the standard dark matter models, the $n=1$ polytrope is used to model dark matter in the form of a Bose-Einstein condensate, namely, an assembly of light individual bosons that acquire a repulsive interaction by occupying the same ground energy state 36 38].

In the present approach to the dark matter halos all the relevant astrophysical quantities can be predicted from the model, and can be directly compared with the corresponding observational parameters (the dark halo mass, the radius of the galaxy, etc), with all the properties of the dark matter halos being determined by a single parameter $\kappa$. Therefore, although lying beyond the range of the present paper, the in-depth comparison of the theoretical predictions of the EiBI gravity model with the galactic and extra galactic scale observations yield tight constraints on the numerical values of $\kappa$, thus leading to a direct viability test of the theory. Work along these lines is currently underway.

\section{Acknowledgments}

FSNL is supported by a Fundação para a Ciência e Tecnologia Investigador FCT Research contract, with reference IF/00859/2012, funded by FCT/MCTES (Portugal). FSNL also acknowledges financial support of the Fundação para a Ciência e Tecnologia through the grants CERN/FP/123615/2011 and CERN/FP/123618/2011. SVS acknowledges financial support of the Russian Foundation for Basic Research through grants No. 11-0201162 and 13-02-12093 . 
[1] M. Aguilar et al. [AMS Collaboration], Phys. Rev. Lett. 110, 141102 (2013).

[2] H. -B. Jin, Y. -L. Wu and Y. -F. Zhou, arXiv:1304.1997 (2013).

[3] J. Binney and S. Tremaine, Galactic dynamics, Princeton University Press, Princeton (1987).

[4] M. Persic, P. Salucci and F. Stel, Month. Not. R. Astron. Soc. 281, 27 (1996).

[5] A. Borriello and P. Salucci, Month. Not. R. Astron. Soc. 323, 285 (2001).

[6] P. Salucci et al., Month. Not. Roy. Astron. Soc. 378, 41 (2007)

[7] H. J. de Vega, P. Salucci, and N. G. Sanchez, New Astronomy 17, 653 (2012).

[8] O. Bertolami, C. G. Boehmer, T. Harko and F. S. N. Lobo, Phys. Rev. D 75, 104016 (2007).

[9] T. Harko and F. S. N. Lobo, Eur. Phys. J. C 70, 373 (2010).

[10] A. S. Eddington, The mathematical theory of relativity, (Cambridge University Press, Cambridge, 1924).

[11] M. Born and L. Infeld, Proc. R. Soc. A 144, 425 (1934).

[12] S. Deser and G. W. Gibbons, Class. Quant. Grav. 15, L35 (1998).

[13] M. Bañados and P. G. Ferreira, Phys. Rev. Lett. 105, 011101 (2010).

[14] T. Delsate and J. Steinhoff, Phys. Rev. Lett. 109, 021101 (2012).

[15] P. Pani, T. Delsate, and V. Cardoso, Phys. Rev. D 85, 084020 (2012).

[16] J. H. C. Scargill, M. Bañados, and P. G. Ferreira, Phys. Rev. D 86, 103533 (2012).

[17] T. Harko, F. S. N. Lobo, M. K. Mak, and S. V. Sushkov, Phys. Rev. D 88, 044032 (2013).

[18] P. Pani and T. P. Sotiriou, Phys. Rev. Lett. 109, 251102 (2012).

[19] P. Pani, V. Cardoso, and T. Delsate, Phys. Rev. Lett. 107, 031101 (2011).

[20] P. P. Avelino, Phys. Rev. D 85, 104053 (2012).
[21] I. L. J. Casanellas, P. Pani, and V. Cardoso, Astrophys. J. 745, 15 (2012).

[22] M. Bouhmadi-Lopez, C.-Y Chen, and P. Chen, arXiv:1302.5013 [gr-qc] (2013).

[23] Y.-H. Sham, L.-M. Lin, and P. T. Leung, Phys. Rev. D 86, 064015 (2012).

[24] Y.-H. Sham, P. T. Leung, L.-M. Lin, Phys. Rev. D 87, 061503(R) (2013).

[25] L. D. Landau and E. M. Lifshitz, The Classical Theory of Fields, Oxford, Pergamon Press (1975).

[26] T. Matos, F. S. Guzman and D. Nunez, Phys. Rev. D 62, 061301 (2000).

[27] U. Nucamendi, M. Salgado and D. Sudarsky, Phys. Rev. D 63, 125016 (2001).

[28] M. K. Mak and T. Harko, Phys. Rev. D 70, 024010 (2004).

[29] C. G. Boehmer, T. Harko, and F. S. N. Lobo, Astropart. Phys. 29, 386 (2008).

[30] T. Harko, Phys. Rev. D 81, 084050 (2010).

[31] P. Salucci and M. Persic, in Dark and visible matter in galaxies, eds. M. Persic and P. Salucci, ASP Conference Series 117, 1 (1997).

[32] S. Chandrasekhar, An introduction to the study of stellar structure, New York, Dover Publications (1957).

[33] G. P. Horedt, Polytropes: applications in astrophysics and related fields, Dordrecht, Boston, Kluwer Academic Publishers (2004).

[34] S. Capozziello and M. De Laurentis, Annalen Phys. 524, 545 (2012).

[35] S. Capozziello, M. De Laurentis, I. De Martino, M. Formisano and S. D. Odintsov, Phys. Rev. D 85, 044022 (2012).

[36] C. G. Boehmer and T. Harko, JCAP 0706, 025 (2007).

[37] T. Harko, Month. Not. Roy. Astron. Soc. 413, 3095 (2011).

[38] T. Harko, JCAP 1105, 022 (2011). 